\title{
LARA, Luis Fernando (2004): Lengua histórica y normatividad, Mexico, El Colegio de México.
}

Lexicographe et métalexicographe de réputation internationale, Luis Fernando Lara a publié de nombreux ouvrages, dictionnaires et articles dans ces domaines. Il réunit d'ailleurs dans ce livre cinq articles publiés récemment dans différentes revues et ouvrages collectifs et touchant le thème de la normativité et ce qui s’y rapproche. Le premier article «Lengua histórica y normatividad» fut publié en 2002 par Eduardo Hopkins dans l'Homenaje a Luis Jaime Cisneros, le deuxième «Normas lingüísticas: pluralidad y jerarquía», dans le numéro 71 (1999) de la revue Español actual. Le troisième «No normas, sino tradiciones», inédit, fait partie d'un hommage de l'Escuela Nacional de Estudios Profesionales (UNAM) de Acatlán à José Moreno de Alba en 2002. Le quatrième «Los diccionarios contemporáneos del español y la normatividad» est un texte qui fut intégré aux actes du Congreso Internacional de la Lengua Española à Valladolid (Espagne) en 2001 et, enfin, le cinquième, «La nueva Ortografia de la Academia», a été publié dans la Nueva Revista de Filología Hispánica en 2001. Le lecteur retrouvera dans cet ouvrage des similitudes avec l'ouvrage El concepto de norma en lingüística (publié en 1976). Lara se situe dans une démarche non structuraliste qui postule que l'apprentissage de la langue se fait dans un contexte de tradition verbale, de valeur de communication et de norme de correction. Lara réfute l'idée que la capacité de parler est uniquement biologique s'appuyant sur une vision réduite de la linguistique moderne, rousseauiste et descriptive c'est-à-dire exempte de références aux contexte social et à la normalisation.

Dans le premier chapitre, il se propose de faire l'analyse de la notion de «langue historique» selon la proposition descriptive de Coseriu. Il postule, en accord avec ce dernier, que c'est par l'existence de la langue historique que l'on reconnaît les particularités des différentes communautés linguistiques et des traditions verbales et non l'inverse. L'auteur s'insurge contre une vision simplificatrice et théorisante de la langue qui évacue la dimension sociale et historique. Il oppose le travail de description de la langue au concept de norme. La linguistique descriptive saussurienne élimine à son avis la question de la langue historique et la normativité. Il précise cependant (43) que cela ne signifie pas pour autant qu'il cherche à contredire Saussure sinon redonner à la complexité historique et sociale de la langue sa pleine valeur.

Le deuxième chapitre intitulé «Normas lingüísticas: pluralidad y jerarquía» aborde notamment la question de l'unité de la langue et la valeur du parlé populaire. Ici l'auteur aborde la question de la normalisation. Il souligne par exemple que «Se puede hacer la distinción entre las normas prescritas por una institución que se arroga la facultad de dictarlas o que se recibe del Estado esa facultad (la Academia, los poderes Ejecutivo o Legislativo, etc.) y las normas socialmente consensadas, que garantizan la unidad de la lengua reconociendo la pluralidad normativa y la variedad de uso» (2004: 60).

L'auteur développe son argumentation autour du thème de la norme et s'appuie pour ce faire essentiellement sur les données du milieu hispanophone comparant l'espagnol du Mexique à celui recommandé par la Real Academia Española. L'auteur réfléchit sur les aspects diatopiques et diastratiques de la norme. Dans le troisième chapitre, le métalexicographe redéfinit la question de la norme dans les variétés de parler espagnol et valorise plutôt les traditions verbales, comme le reflet du consensus linguistique social, empruntant le concept à la linguiste allemande Brigitte Schlieben-Lange, que la norme prescriptive de la Real Academia. 
A cet égard il mentionne, dans le quatrième chapitre intitulé, «Los diccionarios contemporáneos del español y la normatividad» le caractère secondaire qu'ont pris les variétés américaines de l'Espagnol dans le dictionnaire de la Real Academia Espanola.

Lara précise qu'en somme il interprète la norme hispanique à partir de l'unité de la langue et de la reconnaissance des traditions verbales populaires. Cette partie de l'ouvrage porte plus spécifiquement sur les dictionnaires et notamment sur les fonctions des dictionnaires de régionalismes.

Le dernier chapitre s'intéresse à la nouvelle orthographie de la Real Academia. En concluant ce petit ouvrage par ce chapitre Lara nous montre que malgré sa position bien affichée pour la reconnaissance des traditions verbales et des spécificités des variétés d'espagnol américains, il n'en demeure pas moins qu'il reste attentif au fonctionnement politique et correctif de l'instance normative madrilène.

Bref, un ouvrage synthétique qui permet d'entrer rapidement dans la pensée sociolinguistique et lexicographique de Lara et qui reprend les observations qu'il fait sur la langue depuis une trentaine d'années en les actualisant avec de nouveaux travaux. 